\title{
Research progress on the anticancer effects of vitamin K2 (Review)
}

\author{
FAN XV ${ }^{1 *}$, JIEPENG CHEN $^{2 *}$, LILI DUAN ${ }^{2 *}$ and SHUZHUANG LI ${ }^{*}$ \\ ${ }^{1}$ Department of Physiology, Dalian Medical University, Dalian, Liaoning 116044; \\ ${ }^{2}$ Sungen Bioscience Co., Ltd., Shantou, Guangdong 515071, P.R. China
}

Received August 22, 2017; Accepted February 15, 2018

DOI: $10.3892 / \mathrm{ol} .2018 .8502$

\begin{abstract}
Despite the availability of multiple therapeutic methods for patients with cancer, the long-term prognosis is not satisfactory in a number of different cancer types. Vitamin K2 (VK2), which exerts anticancer effects on a number of cancer cell lines, is considered to be a prospective novel agent for the treatment of cancer. The present review aims to summarize the results of studies in which VK2 was administered either to patients with cancer or animals inoculated with cancerous cells, particularly investigating the inhibitory effects of VK2 on cancerous cells, primarily involving cell-cycle arrest, cell differentiation, apoptosis, autophagy and invasion. The present review summarizes evidence stating that treatment with VK2 could positively inhibit the growth of cancer cells, making it a potentially useful approach for the prevention and clinical treatment of cancer. Additionally, the combination treatment of VK2 and established chemotherapeutics may achieve better results, with fewer side effects. Therefore, more attention should be paid to the effects of micronutrients on tumors.
\end{abstract}

Correspondence to: Dr Shuzhuang Li, Department of Physiology, Dalian Medical University, Lv Shun South Road West Section 9, Dalian, Liaoning 116044, P.R. China

E-mail: shuzhuangli@126.com

*Contributed equally

Abbreviations: AML, acute myelocytic leukemia; Bak, Bal-2 antagonist killer 1; Bal-2, B-cell lymphoma 2; Bax, Bcl-2 associated $\mathrm{X}$ protein; Cdk, cyclin-dependent kinase; CL, cardiolipin; CREB, cAMP-response element binding protein; $\mathrm{Cx}$, connexin; $\mathrm{HCC}$, hepatocellular carcinoma; HDGF, hepatoma-derived growth factor; IKK, IкB kinase; MDS, myelodysplastic syndrome; PKD1, protein kinase D1; RXR, retinoid X receptor; RXRE, retinoid X receptor responsive element; SXR, steroid and xenobiotic receptor; VK2, vitamin K2; VK, vitamin K; VK2-O, VK2-2,3 epoxide

Key words: vitamin K2, cancer, drug therapy, cell-cycle arrest, cell differentiation, apoptosis

\section{Contents}

1. Introduction

2. Administration of $\mathrm{VK} 2$ in patients with cancer

3. Anticancer effect of VK2 in animal research

4. Inhibitory effect of VK2 on cancer cells

5. Discussion

\section{Introduction}

Worldwide, cancer is the second-leading cause of mortality following cardiovascular disease. A certain proportion of several cancer types, including colorectal cancer, can only be diagnosed at an advanced stage (1). However, certain cancer types, such as hepatocellular carcinoma (HCC), can easily recur following a short duration despite effective treatment (2). In addition, certain other cancer types are accompanied by severe complications, including failure of the vital organs, despite diagnosis at an early stage and surgery is the contraindication. Established chemotherapies are not suitable for certain patients (1). Hence, the development of a novel therapeutic approach to enhance the overall prognosis of patients with cancer is essential.

Vitamin $\mathrm{K}(\mathrm{VK})$ is an essential lipid-soluble vitamin that is comprised of three types, VK1, VK2, and VK3. VK can activate coagulationfactors(factor II, VII,IX, andX), proteinC and protein $\mathrm{S}$ by facilitating $\gamma$-glutamyl carboxylase to catalyze the carboxylation of glutamic acid residues (3). In addition, VK-dependent $\gamma$-carboxylation has an essential role in maintaining bone homeostasis (4). A lack of VK can lead to severe neonatal bleeding and osteoporosis, which can be treated by the clinical application of VK2 (5).

Previous reports have demonstrated that VK1, VK2 and VK3 can inhibit several neoplastic cell lines at different levels, primarily by inducing apoptosis and cell cycle arrest of cancer cells (6), including HCC, leukemia, colorectal cancer, ovarian cancer, pancreatic cancer and lung cancer. Although the inhibition caused by VK3 is highly potent, VK3 is also highly toxic. By contrast, VK2 is milder, but causes no side effects, whereas VK1 has the least strong function (7). Hence, VK2 is a potential chemotherapeutic candidate for the treatment of cancer. The present review summarizes the results of VK2 against cancer in clinical, animal and in vitro experiments 
and aims to elucidate the mechanisms of anticancer effects of VK2.

\section{Administration of $\mathrm{VK} 2$ in patients with cancer}

To date, several case reports have highlighted the utility of VK2 as a potential antitumor agent. A prior study reported that daily administration of VK2 alleviated pancytopenia in an 80-year-old woman with myelodysplastic syndrome (MDS) and rendered red-cell transfusions redundant after 14 months (8). Similarly, a 72-year-old woman with relapsing acute promyelocytic leukemia was reported to attain complete remission following the combination treatment of VK2 and all-trans retinoic acid (9). Treatment with a combination of VK2 and an angiotensin-converting enzyme inhibitor was shown to shrink a hepatic dysplastic nodule in a 66-year-old Japanese woman with liver cirrhosis (10). Furthermore, the combination of VK2 and vitamin E suppressed the growth of the primary tumor and obliterated the intraperitoneal dissemination in a 65-year-old man with ruptured HCC (11).

These encouraging case reports led to several clinical studies on the anticancer functions of VK2. A multicenter pilot study on VK2 treatment of MDS and post-MDS acute myeloid leukemia (AML) revealed that VK2 could significantly reduce blastic cell numbers in the bone marrow and/or peripheral blood and enhance hematopoiesis, particularly in patients with post-MDS AML (12). Sada et al (13) demonstrated an association between improvements in hematopoiesis and the anti-apoptotic effect of VK2 on normal erythroid progenitors. In addition, the results of several studies (14-18) indicate that VK2 could potentially suppress the development and recurrence of HCC in patients. A study aiming to investigate the VK2-mediated prevention of osteoporosis in female cirrhotic patients deduced that VK2 may decrease the risk of HCC in female cirrhotic patients (14). Another study investigating the function of $\mathrm{VK} 2$ in patients with type $\mathrm{C}$ cirrhosis concluded that VK2 exerted inhibitory effects on HCC development in patients with type $\mathrm{C}$ cirrhosis (15). Mizuta et al (16) reported that VK2 could reduce the recurrence rates of $\mathrm{HCC}$ and enhance the survival rates. Kakizaki et al (17) investigated the effects of VK2 on recurrence in patients with HCC derived from $\mathrm{HCV}$ infection, with the results corroborating those obtained by Mizuta et al (16) Ishizuka et al (18) suggested that VK2 moderately inhibited HCC recurrence following curative hepatectomy. Although the results of certain studies (16-18) did not find statistically significant results, the majority of studies at present, except that conducted by Yoshida et al, considered VK2 as a valuable agent for clinical therapy in patients with cancer. Yoshida et al demonstrated that the VK2-dependent inhibition of HCC recurrence was not proven in a double-blind, randomized, placebo-controlled study (2). However, this study may have had problems with its design. First, Yoshida et al (2) enrolled patients with an increased recurrence and who had recurred after the first treatment. Second, the quality of VK2, which is susceptible to decrease following exposure to light, may also have affected the results. In addition, Zhong et al (19) conducted a meta-analysis based on six recent randomized control trials and one cohort study; the authenticity assessment of this meta-analysis was high. The results indicated that VK2 treatment could significantly decrease the 2- and 3-year tumor recurrence rate, but could not significantly decrease the 1-year recurrence rate, and could also increase the 1-, 2-, and 3-year survival rate (19). Overall, we hypothesize that VK2 can exert positive effects on the therapy of patients with cancer.

\section{Anticancer effect of $\mathrm{VK} 2$ in animal research}

Consistent with the results of clinical studies, data from animal studies indicated that VK2 treatment significantly inhibited tumor growth, without any evident side effects. For instance, exposing male BALB/c-nu/nu mice implanted with PLC/PRF/5 HCC cells to VK2 exhibited evident suppression of the growth of subcutaneous HCC tumors. In addition, decreases in cyclin D1 and cyclin-dependent kinase 4 (CDK4) levels indicated that VK2 may suppress tumor cells in vivo by inducing $G_{1}$ arrest (7). Notably, mice bearing established colorectal cancer cells in the VK2 group exhibited no apparent changes compared with the control group, where the fur and weight of mice changed substantially. Following examination of apoptotic cells in vivo, researchers deduced that VK2 could potentially inhibit colorectal cancer cells by accelerating apoptosis (1). Hence, the induction of the cell-cycle arrest and apoptosis has a crucial role in the antitumor mechanism of VK2. Besides, it is established that VK2 could protect affected cells from forming precancerous lesions to reduce hepatocarcinogenesis in animals (20).

The combination of VK2 with other anticancer agents can be synergistic in tumor-bearing animals. For instance, pretreatment with $\mathrm{VK} 2$ prior to sorafenib treatment is proven to exert more effective HCC growth inhibition in animals than treatment with either alone (21). Similarly, VK2 and phosphatidylcholine together can exert a stronger inhibition on tumorigenesis, which can be applied to prevent hepatocarcinogenesis in patients at a high risk of HCC, particularly those with chronic hepatitis, while preserving the hepatic function (22). To summarize, animal studies have demonstrated that VK2 could repress cancer growth, which is likely to be associated with the induction of cell-cycle arrest and apoptosis.

\section{Inhibitory effect of VK2 on cancer cells}

Several in vitro experiments (1,23-28) have certified the anticancer effect of VK2 against several neoplastic cell lines, including HCC, leukemia, cholangiocarcinoma, ovarian cancer, pancreatic cancer, and colorectal cancer. These studies also investigated the mechanism of VK2 inhibition of cancer cells. Although several details concerning this mechanism require clarification, the results of these studies mainly focus on the growth inhibition of cancer cells caused by the induction of cell-cycle arrest, cell differentiation, apoptosis and autophagy, and the suppression of cancer cell invasion.

Inhibition of proliferation of cancer cells by VK2. VK2 can inhibit the proliferation of cancer cells by inducing the cell-cycle arrest of cancer cells, in which inhibition of nuclear factor- $\kappa \mathrm{B}(\mathrm{NF}-\kappa \mathrm{B})$ activity has a crucial role. NF- $\kappa \mathrm{B}$ is a regulatory factor that can be simulated by cytokines to participate in the immune and inflammatory reaction (29). In addition, as a nuclear transcription factor, $\mathrm{NF}-\kappa \mathrm{B}$ is associated with cell growth by regulating the cyclin D1 gene (23). Cyclin D1 can 
contribute to the $\mathrm{G}_{1}-\mathrm{S}$ transformation during the cell cycle by binding to CDK4 or CDK6 (30). Reportedly, NF- $\mathrm{kB}$ and cyclin D1 are involved in carcinogenesis. In cancer cells, VK2 can downregulate the expression of cyclin D1 by inhibiting the binding of NF- $\mathrm{kB}$ to the cyclin D1 promoter, which is followed by cell-cycle arrest in the $\mathrm{G}_{1}$ phase (23). Regarding the suppression of the aberrant activity of NF- $\mathrm{KB}$ by VK2, certain studies reported results using HCC cells as follows. First, VK2

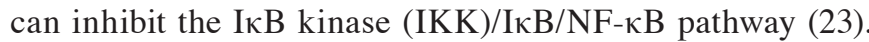
Usually, NF- $\kappa \mathrm{B}$ exists in the cytosol and is inactivated by the

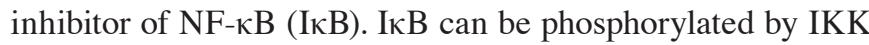
in response to certain stimulatory factors prior to degrading $\mathrm{I} \kappa \mathrm{B}$, which leads to the nuclear translocation of NF- $\mathrm{BB}$ and the activation of associated genes regulated by NF- $\mathrm{kB}$ (31). VK2 inhibited the function of IKK, which suppressed the phosphorylation of IkB and activity of NF-kB (23). Second, VK2 can inhibit the protein kinase $\mathrm{C} \alpha(\mathrm{PKC} \alpha) / \mathrm{NF}-\kappa \mathrm{B}$ and $\mathrm{PKC} \varepsilon /$ protein kinase D1 (PKD1)/NF- $\mathrm{KB}$ pathways (24). Protein kinase $\mathrm{C}(\mathrm{PKC})$ is a phospholipid-dependent kinase family, which is reportedly involved in the activation of NF-kB and cell growth. PKD1 is the effector of PKC and has been confirmed to be phosphorylated entirely by PKCE (32). Xia et al (24) suggested that VK2 inhibited NF-кB activation by inhibiting the catalytic action of activated PKC $\alpha$, not by phosphorylation of $\mathrm{PKC} \alpha$, and that VK2 could also inhibit NF- $\mathrm{KB}$ activation by hindering the phosphorylation of $\mathrm{PKC \varepsilon}$ and thus further suppressing PKD1 phosphorylation. Besides, PKD1 was found to promote I $\kappa$ B phosphorylation, from which the PKC/PKD1 pathway may be one of the upstream pathways through which VK2 restrains IKK activity.

In addition to PKC, protein kinase $\mathrm{A}$ (PKA), which can lead to cell-cycle arrest at the $\mathrm{G}_{1}$ and $\mathrm{G}_{2}-\mathrm{M}$ phase, is another type of kinase involved in the mechanism of VK2 against tumor cells. VK2 has been identified to stimulate the phosphorylation of PKA and activate activating protein 2 (AP-2), upstream transcription factor-1 (USF-1), and cAMP-response element binding protein (CREB) transcriptional factors to inhibit the proliferation of HCC cells (33). At present, the activities of these transcriptional factors are possibly the downstream pathways of VK2 activating PKA, because PKA is the only known regulator of AP-2, USF-1, and CREB. However, the details about the mechanism by which VK2 activates PKA continue to require clarification.

The promotion of VK2 on the transcription of p21 and p27 is another way to effectively promote cell-cycle arrest of cancer cells. Cell-cycle regulatory proteins p27 and p21 are the two members of the Cip/Kip family, working as CDK inhibitors, which have a negative role in the cell-cycle progression of the $\mathrm{G}_{1}-\mathrm{S}$ transition. In HCC cells, VK2 induces $\mathrm{G}_{1}$ arrest by activating the promoter of the $\mathrm{p} 21$ gene and increasing the expression of $\mathrm{p} 21$; however, the activity of $\mathrm{p} 27$ is not interfered with by VK2. It has been identified that the primary interaction sites of VK2 and the promoter region of the p21 gene are the sequences between $-2,130$ and $-102 \mathrm{bp}$ of p21 (34). By contrast, VK2 upregulates the expression of p27 to result in the $\mathrm{G}_{0}-\mathrm{G}_{1}$ arrest in leukemic cells (28). The difference between VK2 regulating p21 and p27 in HCC cells and leukemic cells is likely attributed to cancer cell types, and the role p21 and p27 have in other tumor cell lines inhibited by VK2 warrants further investigation.
Furthermore, suppression of the c-MYC expression is possibly associated with the cell-cycle arrest of cancer cells induced by VK2. c-MYC is highly expressed in a variety of human tumors, and the induction of cell-cycle arrest and differentiation of AML cells by all-trans retinoic acid is primarily attributed to c-MYC downregulation (35). Maniwa et al (35) identified VK2 suppression on c-MYC expression and deduced that $5 \mu \mathrm{M}$ VK2 exposure inhibited c-MYC expression in HL-60 leukemia cells to $~ 80 \%$ that of control cells.

Finally, in HCC cells, it has been identified that VK2 could evidently downregulate the expression of hepatoma-derived growth factor (HDGF) by interfering in the initiation of HDGF gene transcription and subsequently inhibiting the proliferation of cancer cells. The region -1 to $-150 \mathrm{bp}$ in the promoter of the HDGF gene was detected to be the binding site of VK2 (36). HDGF is highly expressed in various cancer cells and can transmit a proliferative signal to cells in the early $\mathrm{G}_{1}$ phase and then stimulate the increase of cyclin D (36). Hence, the inhibitory activity of VK2 on HDGF may lead to $\mathrm{G}_{1}$ arrest and then suppress the cell growth. There are two problems that remain to be solved: i) Which pathways exert functions in the regulation of VK2 to HDGF; and ii) whether VK2 could suppress cancer cell growth by downregulating other growth factors (Fig. 1).

Induction of cell differentiation in cancer cells by VK2. It has been certified in various cell lines that cell-cycle arrest is closely associated with cell differentiation. VK2 can exhibit differentiation-inducing results by the induction of the $\mathrm{G}_{0}-\mathrm{G}_{1}$ arrest in cancer cells. For instance, Miyazawa et al (28) reported that VK2 treatment induced monocytic differentiation in HL-60-Bcl-2 leukemia cells, with an evident increase in the proportion of cells in the $\mathrm{G}_{0}-\mathrm{G}_{1}$ phase. Maniwa et al (35) determined that VK2 reduced c-MYC expression, which subsequently contributed to cell growth arrest and cell differentiation. Hence, the molecular activities regulated by VK2, including the induction of cell-cycle arrest in cancer cells, are likely to be crucial to the mechanism of the VK2-dependent stimulation of cancer cell differentiation. Notably, cell-cycle arrest is only one of the processes that can lead to the differentiation of cancer cells.

VK2 suppresses connexin 43 (Cx43) expression and enhances Cx32 activity, which is another mechanism of cancer cell differentiation. Cx proteins are comprised of gap junction channel structures that mediate intercellular communication to maintain tissue homeostasis. Studies indicate that $\mathrm{Cx}$ genes have tumor-suppressing effects and the distribution of $\mathrm{Cx}$ is tissue specific (37). Cx32 is mainly expressed in hepatocytes. The risk of tumor development can be raised when knocking out the Cx32 gene in mice. However, Cx43 is not found in regular hepatocytes, and its expression is highly upregulated in HCC cells (38). Regarding cell differentiation induced by VK2, Kaneda et al (37) reported that VK2 exposure could drive Huh7 HCC cells to adopt a normal liver cell phenotype by upregulating $\mathrm{Cx} 32$ indirectly via downregulation of $\mathrm{Cx} 43$ at the transcriptional level, and the increase of gap junction intercellular communication enhanced by $\mathrm{VK} 2$ is potentially due to $\mathrm{Cx} 32$ upregulation.

VK2-induced differentiation is partly associated with steroid and xenobiotic receptor (SXR). VK2 can work as the 


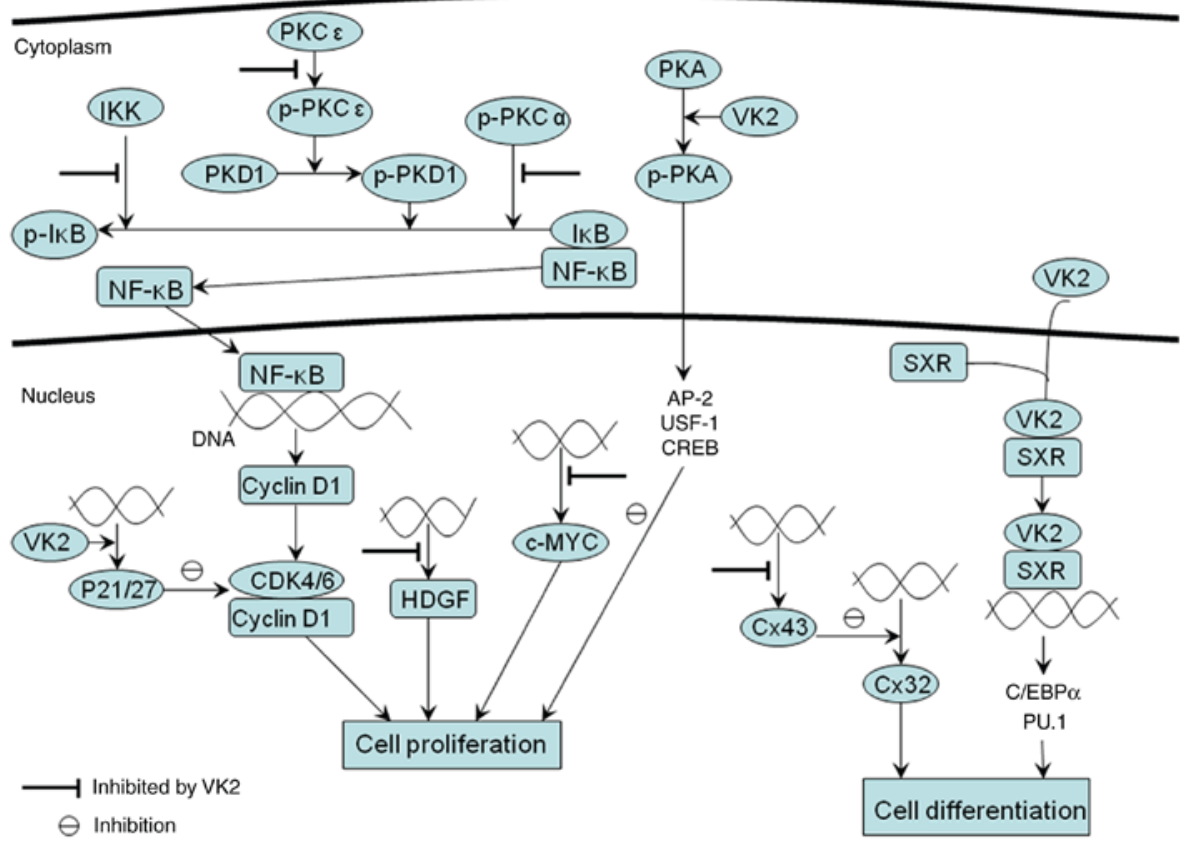

Figure 1. Inhibition of the proliferation of cancer cells by VK2 and induction of cell differentiation in cancer cells by VK2. VK2 inhibits the proliferation of cancer cells by inducing cell-cycle arrest. In cancer cells, IKB is phosphorylated by IKK, PKD1 or p-PKC $\alpha$, followed by the nuclear translocation of NF-kB and the activation of cyclin D1 genes regulated by NF- $\mathrm{KB}$, which enhances the expression of cyclin $\mathrm{D} 1$ and its binding to CDK4/6, promoting the proliferation of cancer cells. PKD1 is phosphorylated entirely by p-PKCE. Results of studies using HCC cells revealed that VK2 could inhibit the function of IKK, the phosphorylation of PKCE and the catalytic action of activated PKC $\alpha$, but not the phosphorylation of PKC $\alpha$. This suppresses the aberrant activity of NF- $\mathrm{KB}$ and induces cell-cycle arrest in cancer cells. The cell-cycle regulatory proteins p27 and p21 are CDK inhibitors, acting to hinder cell-cycle progression. In HCC cells, VK2 increases the expression of P21 and then leads to cell cycle arrest. However, in leukemic cells, VK2 upregulates the expression p27 not p21 to induce cell cycle arrest. Besides, VK2 suppresses the high expression of HDGF in HCC cells and c-MYC in HL-60 leukemia cells at the transcriptional level, and then induces cell-cycle arrest. VK2 has been identified to stimulate the phosphorylation of PKA and activate AP-2, USF-1 and CREB transcriptional factors to inhibit the proliferation of HCC cells. At present, the activities of these transcriptional factors are potential downstream pathways of VK2 activation of PKA. In HCC cells, Cx43 expression is highly upregulated, which inhibits Cx32 expression. The VK2-dependent suppression of Cx43 expression at the transcriptional level enhances Cx32 activity, altering cancer cells differentiation. In addition, VK2 promotes differentiation of myeloid progenitors partly due to its binding to SXR and upregulation of transcriptional factors C/EBP $\alpha$ and PU.1 crucial for myeloid development. VK2 binding to SXR may subsequently improve the expression of $\mathrm{C} / \mathrm{EBP} \alpha$ and PU.1, which may elucidate the reason behind the therapeutic effect of VK2 on patients with MDS. NF- $\mathrm{kB}$, nuclear

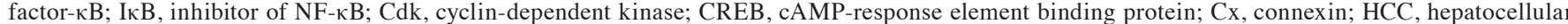
carcinoma; HDGF, hepatoma-derived growth factor; IKK, IкB kinase; MDS, myelodysplastic syndrome; p-PKA, phosphorylated protein kinase A; p-PKC $\alpha$, phosphorylated PKC $\alpha$; p-PKC $\varepsilon$, phosphorylated PKC $\varepsilon$; PKD1, protein kinase D1; p-PKD1, phosphorylated protein kinase D1; SXR, steroid and xenobiotic receptor; VK2, vitamin K2; AP-2, activating protein2; USF-1, upstream transcription factor; C/EBP $\alpha$, CCAAT/enhancer-binding protein- $\alpha$.

ligand of SXR, a nuclear receptor, and their binding largely exerts osteoprotective function. Sada et al (13) investigated the effects of VK2 on normal hematopoietic progenitor cells and revealed that VK2 promotes the differentiation of myeloid progenitors partly owing to its binding to SXR and the upregulation of the transcriptional factors CCAAT/enhancer-binding protein $\alpha(\mathrm{C} / \mathrm{EBP} \alpha)$ and PU.1, which are crucial for myeloid development. In addition, VK2 binding to SXR may subsequently improve the expression of $\mathrm{C} / \mathrm{EBP} \alpha$ and PU.1, which may aid elucidation of the therapeutic effect of VK2 on patients with MDS (Fig. 1) (13).

Induction of apoptosis of cancer cells by VK2. The mitochondrial pathway is a crucial process through which VK2 exerts its pro-apoptotic function. Following treatment with VK2, the mitochondrial membrane potential is depolarized and cytochrome $\mathrm{c}$ is released from the mitochondria into the cytosol to form the apoptosome, driving activation of caspase-9, which ultimately leads to the activation of caspase- 3 and initiation of cell apoptosis (39-41). It is anticipated that the selective binding of VK2 and VK2-2,3 epoxide (VK2-O) to the mitochondrial protein $\mathrm{Bcl}-2$ antagonist killer 1 (Bak) is an essential molecular mechanism of VK2 dissipating the mitochondrial membrane potential of leukemia cells. In addition, the direct binding of VK2 to Bak specifically resulted in a post-translational modification of Bak (39). It has been revealed that VK2 and VK2-O metabolized from VK2 could non-covalently bind to Bak through the Arg-169 and Trp-170 residues, and that VK2-O further covalently attached to the Cys-166 residue of Bak in HL-60 leukemia cells (39). In addition, VK2 treatment increased the intracellular level of the reactive oxygen species (ROS) $(39,40)$. Evidence indicates that VK2-induced ROS generation occurred prior to the induction of apoptosis. Assumedly, ROS contributes by converting VK2 to VK2-O (39). Besides Bak, other Bal-2 family members also have crucial roles in the mitochondrial apoptosis pathway induced by VK2. VK2 decreases Bcl-2 expression and increases the expression of Bcl-2-associated X protein (Bax) in a newly established MDS cell line, which was associated with apoptosis (42). Furthermore, Tsujioka et al (41) determined that the ratio of B-cell lymphoma (Bcl)-extra large and Bcl-extra small expression decreased when exposing myeloma cells to $10 \mu \mathrm{M}$ VK2; however, the expression of Bcl-2 and Bax remained unaffected. VK2 can thus alter the expression 
of the Bcl-2 family, tending to a pro-apoptotic balance and activating the mitochondrial apoptosis pathway of cancer cells. It is hypothesized that the release of cytochrome $\mathrm{c}$ from the mitochondria partly results from the acidic phospholipid cardiolipin (CL), abundant in the outer membrane of the mitochondria, being peroxidated by ROS (40), which remains to be confirmed.

Furthermore, the mitogen-activated protein kinase (MAPK) pathway is essential for the VK2-mediated mitochondrial apoptosis pathway $(25,26,41,43)$. In the MAPK superfamily, p38 MAPK and c-Jun N-terminal kinase (JNK) pathways respond to stress and contribute to inflammation or even apoptosis, whereas the extracellular signal-related kinase (ERK) pathway reacts to growth factors or other external mitogenic signals by stimulating cell proliferation and resisting apoptotic signals. VK2 activates p38 MAPK to its phosphorylated form and subsequently results in apoptosis of the neoplastic cells $(41,44)$. Tsujioka et al $(41)$ identified this in myeloma cells, following which they detected that the mitochondrial membrane potential was depolarized and caspase- 9 was activated, indicating that the phosphorylation of p38 MAPK stimulated by VK2 possibly induces apoptosis by initiating the mitochondrial pathway. In addition, these authors found that ROS generation stimulated by VK2 is associated with this apoptosis (41). Sibayama-Imazu et al (25) investigated the apoptosis induction of PA-1 ovarian cancer cells by VK2 and concluded that the increase of synthesis and accumulation in the mitochondria of TR3, also known as Nur77 and neuron growth factor inducible factor I-B, highly expressed in various tumor cell lines, is possibly associated with the mitochondrial apoptosis pathway induced by VK2. In addition, Sibayama-Imazu et al (25) deduced that VK2 may activate JNK to phosphorylate TR3 and increase TR3 levels in the mitochondria. VK2 is reported to inhibit ERK phosphorylation by suppressing Ras activation and subsequently suppressing the activation of MAPK kinase (MEK), which causes the apoptosis of HCC cells (43). Conversely, research investigating the inhibitory role of $\mathrm{VK} 2$ on pancreatic cancer demonstrated that apoptosis of cancer cells treated with VK2 was primarily associated with an increase in phosphorylated ERK (26). This contradiction may be due to differences in the type of cancer cells, and requires further investigation. Another unsolved problem is that not all pancreatic cancer cell lines are sensitive to VK2 treatment. Hence, the effects of VK2 on pancreatic cancer cells warrant further investigation.

In addition to the mitochondrial pathway, described as an intrinsic pathway, the extrinsic apoptosis pathway also participates in the mechanism of VK2-dependent induction of cell death. Evidence indicates that VK2 can induce apoptosis in cancer cells by activating p53 and initiating the extrinsic apoptosis pathway (45). Notably, p53 is a multi-faceted tumor-repressor gene capable of inducing cell-cycle arrest, cell differentiation or apoptosis in reaction to oncogenic stress (46). The extrinsic apoptosis pathway is depedent on the death receptors binding to ligands to form the death-inducing signaling complex, which then contributes to caspase- 8 activation and further activates caspase-3 (47). A study investigating the antitumor effects of VK2 in Smmc-7721 HCC cells established that VK2 stimulated the extrinsic apoptosis pathway by increasing p53 phosphorylation and then activating caspase-8 (Fig. 2) (45).

Another previous study (6) reported that a large dose of VK2 could induce apoptosis of Hep40 HCC cells by increasing the expression of c-JUN and c-MYC; however, it did not identify the detailed apoptosis pathway.

Induction of autophagy in cancer cells by VK2. In different cancer cell lines, VK2 can inhibit the growth of cancer cells by evoking autophagy. Autophagy is a mechanism that aids cell survival in response to stresses, such as nutrition starvation. It has been reported that autophagy is essential for the inhibition of certain antitumor agents in cancer $(27,48)$. Owing to a lack of clarity regarding the molecular mechanism that lead to autophagy, whether autophagy protects or promotes cell death is debatable. Yokoyama et al (48) reported that VK2 could stimulate apoptosis and autophagy in leukemic cells simultaneously, but autophagy is more dominant when Bcl-2 is highly expressed, restraining apoptosis. Hence, Yokoyama et al (48) suggested that autophagy may act as an alternative inducer of apoptosis. Another study that exposed cholangiocellular carcinoma cell lines to VK2 identified the effects of inducing apoptosis and cell-cycle arrest to be inconspicuous; however, the autophagy induction exerted a maximal effect in VK2 inhibiting cholangiocellular carcinoma cells (27). Among these cells lines, the TFK-1 cell line exhibited a higher expression of $\mathrm{Bcl}-2$. On the basis of these two studies, it can be inferred that the molecular mechanism of apoptosis may lead to autophagy, which could be regarded as the cell state prior to apoptosis. In addition, at least in cholangiocellular carcinoma cells, Bcl-2 may be involved in the determination of whether cells eventually undergo apoptosis or autophagy. When Bcl-2 is highly expressed, apoptosis induction may change to autophagy. In short, inducing autophagy is a significant part in the mechanism of VK2-dependent inhibition of cancer cells, although further details remain to be investigated.

Invasion-inhibiting functions of VK2 in cancer cells. On the basis of the study conducted by Ide et al (49), VK2 can restrain the invasion of tumor cells mainly by downregulating the expression of matrix metalloproteinases (MMPs) at the transcriptional level. MMPs are a group of proteinases that degrade extracellular matrix proteins, which are reportedly linked to tumor invasion and metastasis; AP-1 and NF- $\kappa$ B are the common transcription factors of the promoter regions of MMPs. AP-1 is the transcription complex comprising proto-oncogene proteins c-FOS and c-JUN, and activity of the MAPK pathway leads to the phosphorylation of specific threonine and tyrosine residues of c-FOS and c-JUN (50). Ide et al (49) revealed that VK2 could inhibit MMP expression by mediating NF- $\mathrm{B}$ inhibition and downregulating AP-1 by suppressing the ERK and JNK pathways, which restrained the invasion of HCC cells.

Synergistic effect of VK2 in combination with other chemotherapeutics. In several cases, the combination of VK2 with other chemotherapy agents can produce stronger effects than the use of either alone. The mechanisms associated with the synergistic effects can also be classified into induction of the cell-cycle arrest, differentiation, and apoptosis in cancer 


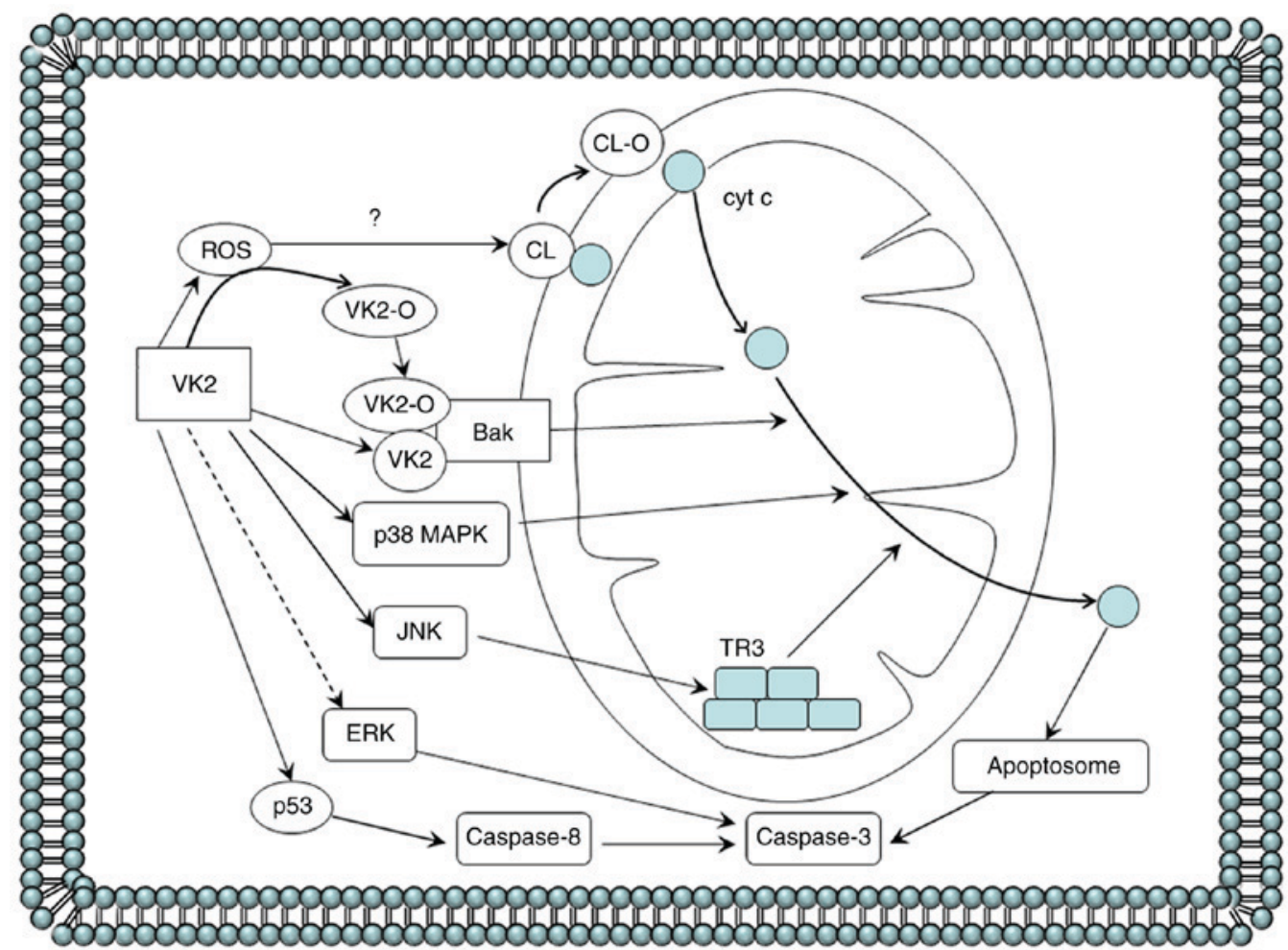

Figure 2. Cell apoptosis induced by VK2 in cancer cells. VK2 induces apoptosis in cancer cells by depolarizing the mitochondrial membrane potential, followed by cytochrome $\mathrm{c}$ release from the mitochondria into the cytosol to form apoptosomes, which then activates caspase-3. The precise mechanism of VK2-dependent initiation of the mitochondrial apoptosis pathway of cancer cells is as follows. In HL-60 leukemia cells, VK2 and VK2-O selectively binds to the mitochondrial protein Bak. VK2-induced ROS generation prior to the induction of apoptosis possibly contributes by converting VK2 to VK2-O. In myeloma cells, VK2 activates p38 MAPK to its phosphorylated form. VK2 exposure in PA-1 ovarian cancer cells may activate JNK to phosphorylate TR3, also known as Nur77 and neuron growth factor inducible factor I-B, and increase TR3 levels in the mitochondria. The hypothesis that the release of cytochrome $\mathrm{c}$ from the mitochondria partly results from the acidic phospholipid CL being peroxidated by ROS is yet to be confirmed. The role of ERK in the VK2-dependent activation of caspase- 3 and induction of apoptosis in hepatocellular carcinoma and pancreatic cancer cells is contradictory, so this pathway is represented with a dashed line. VK2 can inhibit ERK phosphorylation by suppressing the Ras activation and subsequently suppressing the catalysis of MEK, which causes apoptosis in HCC cells. Conversely, VK2-dependent induction of pancreatic cancer cell apoptosis is primarily associated with an increase in levels of phosphorylated ERK. In addition, VK2 stimulates the extrinsic apoptosis pathway by increasing p53 phosphorylation and then activating caspase-8 in Smmc-7721 HCC cells. Bak, Bcl-2 antagonist killer 1; Bcl-2, B-cell lymphoma 2; Bax, Bcl-2 associated X protein; CL, cardiolipin; HCC, hepatocellular carcinoma; VK2, vitamin K2; VK2-O, VK2-2,3 epoxide; ROS, reactive oxygen species; MAPK, mitogen-activated protein kinase; JNK, c-Jun N-terminal kinase; MEK, MAPK kinase; ERK, extracellular-signal-related kinase.

cells; however, the specific details may slightly vary from the mechanism of action of VK2 alone. VK2 pretreatment can restrain NF-kB activation and increase cyclin D1 expression caused by 5-fluorouracil (5-FU), promoting cell-cycle arrest and improving the 5-FU-dependent inhibition of HCC cell growth (51). In addition, VK2 can enhance the ability of cotylenin A to induce cell differentiation in HL-60 leukemia cells, as VK2 can enhance the increase of cyclin G2 expression stimulated by cotylenin A (35). Cyclin G2 plays a beneficial role in promoting and maintaining cell-cycle arrest, but treatment with VK2 alone induces a non-significant cyclin G2 expression in cancer cells (35). When treating with VK2 and sorafenib together in HCC cells, VK2 can counteract the decrease of p21 level and improve the inhibition of ERK phosphorylation induced by sorafenib, which promotes cell-cycle arrest and apoptosis (21).

VK2 can augment the efficacy of retinoids in cancer cells $(9,43)$. Retinoids suppress cell growth through nuclear receptor retinoid $X$ receptor ( $R X R)$, which can bind to the $\mathrm{RXR}$-responsive element located in the promoter regions of retinoid-target genes. In HCC cells, the Ras/ERK pathway is aberrantly activated, which causes accumulation of phosphorylated $\mathrm{RXR} \alpha$ and weakens the effects of retinoids (43). However, treatment with VK2 plus retinoid can apparently decrease the level of phosphorylated ERK and phosphorylated $\mathrm{RXR} \alpha$ by cooperatively inhibiting the Ras/ERK pathway, which contributes to retinoid binding of functional RXR $\alpha$ and to the apoptosis of HCC cells (43). This mechanism is likely to be the reason why the combination of acyclic retinoid and VK2 could decrease the recurrence rate of HCC in clincal trials. Therefore, VK2 alone and the combination of VK2 with other antitumor agents requires further investigation.

Vitamin D3 is another micronutrient capable of restricting the growth of cancer cells by inhibiting proliferation and stimulating differentiation (52). However, an adverse effect of vitamin D3 treatment is hypercalcemia, which can lead to vascular calcification. VK2 regulates the calcium deposition between bone tissue and other tissues, and inhibits the formation of vascular calcified foci. The combination of vitamin D3 with VK2 on cancer cells can synergistically improve the induction of cellular differentiation and also significantly reduces the risk of hypercalcemia and vascular calcification $(52,53)$. 


\section{Discussion}

The present review summarizes the effects of VK2 on cancer in clinical, in vivo, and in vitro studies. Clinical trials demonstrated that VK2 has the potential to improve the prognosis of patients with cancer. In addition, evidence indicates that VK2 treatment can prevent HCC in patients with hepatic cirrhosis, and the dietary intake of VK2 can decrease the risk of developing cancer, particularly prostate and lung cancer (54). Furthermore, VK2 is confirmed to restrain tumor cell growth in animal studies $(1,7,20-22,55)$, with cell-cycle arrest and apoptosis involved in this inhibition. In vitro studies $(1,23-27,35)$ certified that VK2 could inhibit the growth of several cancer cell lines. Although several detailed links remain to be investigated, studies included in the present review $(23-25,33-37,39,41,45,48,49)$ indicated that induction of the cell-cycle arrest, cell differentiation, apoptosis, and autophagy is crucial for VK2-dependent suppression of cancer cell growth. Certain protein kinases, such as PKA and PKC, signaling pathways, such as the MAPK pathways, transcription factors, such as NF- $\mathrm{kB}$ and $\mathrm{AP}-2$, and essential proteins, such as Bak and Cx43, are involved in the mechanism of VK2 activity against cancer cells $(23,24,33,37,41)$. The combination treatment of VK2 with other chemotherapeutics, such as sorafenib, can exert a synergistic effect and reduce adverse drug reactions.

In conclusion, VK2 can positively inhibit cancer cells. VK2 appears to be an extremely promising agent with very limited toxicity, which can be a useful option for prevention of cancer and clinical therapy of cancer. However, the inhibition of vitamin K and D in cancers indicated that vitamins might have positive effects on the prevention and therapy of tumors. Therefore, the effects of vitamins or minerals on tumors should be investigated further.

\section{Acknowledgements}

Not applicable.

\section{Funding}

The present study was funded by the National Nature Science Foundation of China (grant no. 30971065), the Science and Technology Plan of Dalian (grant no. 2012E12SF074) and the Education Fund Item of Liaoning Province (grant no. 2009 A 194).

\section{Availability of data and materials}

All data analyzed during this study are included in this published article.

\section{Authors' contributions}

SL decided the topic of the manuscript. FX was a major contributor in writing the manuscript. SL, FX, JC and LD revised it critically for important intellectual content. JC and LD analyzed and interpreted all the data. All authors read and approved the final manuscript.

\section{Ethics approval and consent to participate}

Not applicable.

\section{Consent for publication}

All authors have read and approved the final manuscript.

\section{Competing interests}

The authors declare that they have no competing interests.

\section{References}

1. Ogawa M, Nakai S, Deguchi A, Nonomura T, Masaki T, Uchida N, Yoshiji H and Kuriyama S: Vitamins K2, K3 and K5 exert antitumor effects on established colorectal cancer in mice by inducing apoptotic death of tumor cells. Int J Oncol 31: 323-331, 2007.

2. Yoshida H, Shiratori Y, Kudo M, Shiina S, Mizuta T, Kojiro M Yamamoto K, Koike Y, Saito K, Koyanagi N, et al: Effect of vitamin $\mathrm{K} 2$ on the recurrence of hepatocellular carcinoma. Hepatology 54: 532-540, 2011.

3. Li ZQ, He FY, Stehle CJ, Wang Z, Kar S, Finn FM and Carr BI: Vitamin K uptake in hepatocytes and hepatoma cells. Life Sci 70: 2085-2100, 2002.

4. Iwamoto J, Takeda T and Sato Y: Effects of vitamin K2 on osteoporosis. Curr Pharm Des 10: 2557-2576, 2004.

5. Ushiroyama T, Ikeda A and Ueki M: Effect of continuous combined therapy with vitamin $\mathrm{K}(2)$ and vitamin $\mathrm{D}(3)$ on bone mineral density and coagulofibrinolysis function in postmenopausal women. Maturitas 41: 211-221, 2002.

6. Bouzahzah B, Nishikawa Y, Simon D and Carr BI: Growth control and gene expression in a new hepatocellular carcinoma cell line, Hep40: Inhibitory actions of vitamin K. J Cell Physiol 165: 459-467, 1995.

7. Hitomi M, Yokoyama F, Kita Y, Nonomura T, Masaki T, Yoshiji $\mathrm{H}$, Inoue H, Kinekawa F, Kurokohchi K, Uchida N, et al: Antitumor effects of vitamins K1, K2 and K3 on hepatocellular carcinoma in vitro and in vivo. Int J Oncol 26: 713-720, 2005.

8. Takami A, Nakao S, Ontachi Y, Yamauchi H and Matsuda T: Successful therapy of myelodysplastic syndrome with menatetrenone, a vitamin K2 analog. Int J Hematol 69: 24-26, 1999.

9. Fujita $\mathrm{H}$, Tomiyama $\mathrm{J}$ and Tanaka $\mathrm{T}$ : Vitamin $\mathrm{K} 2$ combined with all-trans retinoic acid induced complete remission of relapsing acute promyelocytic leukaemia. Br J Haematol 103: 584-585, 1998.

10. Yoshiji H, Noguchi R, Yamazaki M, Ikenaka Y, Sawai M, Ishikawa M, Kawaratani H, Mashitani T, Kitade M, Kaji K, et al: Combined treatment of vitamin K2 and angiotensin-converting enzyme inhibitor ameliorates hepatic dysplastic nodule in a patient with liver cirrhosis. World J Gastroenterol 13: 3259-3261, 2007.

11. Otsuka T, Hagiwara S, Tojima H, Yoshida H, Takahashi T, Nagasaka K, Tomioka S, Ando T, Takeuchi K, Kori T, et al: Hepatocellular carcinoma with peritoneal dissemination which was regressed during vitamin $\mathrm{K} 2$ and vitamin $\mathrm{E}$ administration. Intern Med 46: 711-715, 2007.

12. Miyazawa K, Nishimaki J, Ohyashiki K, Enomoto S, Kuriya S, Fukuda R, Hotta T, Teramura M, Mizoguchi H, Uchiyama $\mathrm{T}$ and Omine M: Vitamin K2 therapy for myelodysplastic syndromes (MDS) and post-MDS acute myeloid leukemia: Information through a questionnaire survey of multi-center pilot studies in Japan. Leukemia 14: 1156-1157, 2000.

13. Sada E, Abe Y, Ohba R, Tachikawa Y, Nagasawa E, Shiratsuchi M and Takayanagi R: Vitamin K2 modulates differentiation and apoptosis of both myeloid and erythroid lineages. Eur J Haematol 85: 538-548, 2010.

14. Habu D, Shiomi S, Tamori A, Takeda T, Tanaka T, Kubo S and Nishiguchi S: Role of vitamin K2 in the development of hepatocellular carcinoma in women with viral cirrhosis of the liver. JAMA 292: 358-361, 2004. 
15. Kojima K, Tamano M, Akima T, Hashimoto T, Kuniyoshi T, Maeda C, Majima Y, Kusano K, Murohisa T, Iijima M and Hiraishi H: Effect of vitamin K2 on the development of hepatocellular carcinoma in type C cirrhosis. Hepatogastroenterology 57: 1264-1267, 2010

16. Mizuta T, Ozaki I, Eguchi Y, Yasutake T, Kawazoe S, Fujimoto K and Yamamoto K: The effect of menatetrenone, a vitamin K2 analog, on disease recurrence and survival in patients with hepatocellular carcinoma after curative treatment: A pilot study. Cancer 106: 867-872, 2006

17. Kakizaki S, Sohara N, Sato K, Suzuki H, Yanagisawa M, Nakajima H, Takagi H, Naganuma A, Otsuka T, Takahashi $\mathrm{H}$, et al: Preventive effects of vitamin $\mathrm{K}$ on recurrent disease in patients with hepatocellular carcinoma arising from hepatitis C viral infection. J Gastroenterol Hepatol 22: 518-522, 2007.

18. Ishizuka M, Kubota K, Shimoda M, Kita J, Kato M, Park KH and Shiraki T: Effect of menatetrenone, a vitamin $\mathrm{k} 2$ analog, on recurrence of hepatocellular carcinoma after surgical resection: A prospective randomized controlled trial. Anticancer Res 32: 5415-5420, 2012

19. Zhong JH, Mo XS, Xiang BD, Yuan WP, Jiang JF, Xie GS and Li LQ: Postoperative use of the chemopreventive vitamin K2 analog in patients with hepatocellular carcinoma. PLoS One 8: $58082,2013$.

20. Yoshiji H, Kuriyama S, Noguchi R, Yoshii J, Ikenaka Y, Yanase K, Namisaki T, Kitade M, Yamazaki M, Masaki T and Fukui H: Combination of vitamin K2 and the angiotensin-converting enzyme inhibitor, perindopril, attenuates the liver enzyme-altered preneoplastic lesions in rats via angiogenesis suppression. J Hepatol 42: 687-693, 2005.

21. Zhang Y, Zhang B, Zhang A, Zhao Y, Zhao J, Liu J, Gao J, Fang D and Rao Z: Synergistic growth inhibition by sorafenib and vitamin $\mathrm{K} 2$ in human hepatocellular carcinoma cells. Clinics 67: 1093-1099, 2012.

22. Sakakima Y, Hayakawa A, Nagasaka T and Nakao A: Prevention of hepatocarcinogenesis with phosphatidylcholine and menaquinone-4: In vitro and in vivo experiments. J Hepatol 47: 83-92, 2007.

23. Ozaki I, Zhang H, Mizuta T, Ide Y, Eguchi Y, Yasutake T, Sakamaki T, Pestell RG and Yamamoto K: Menatetrenone, a vitamin K2 analogue, inhibits hepatocellular carcinoma cell growth by suppressing cyclin D1 expression through inhibition of nuclear factor kappaB activation. Clin Cancer Res 13: 2236-2245, 2007.

24. Xia J, Matsuhashi S, Hamajima H, Iwane S, Takahashi $H$, Eguchi Y, Mizuta T, Fujimoto K, Kuroda S and Ozaki I: The role of PKC isoforms in the inhibition of NF-kappaB activation by vitamin K2 in human hepatocellular carcinoma cells. J Nutr Biochem 23: 1668-1675, 2012.

25. Sibayama-Imazu T, Fujisawa Y, Masuda Y, Aiuchi T, Nakajo S, Itabe $\mathrm{H}$ and Nakaya K: Induction of apoptosis in PA-1 ovarian cancer cells by vitamin $\mathrm{K} 2$ is associated with an increase in the level of TR3/Nur77 and its accumulation in mitochondria and nuclei. J Cancer Res Clin Oncol 134: 803-812, 2008.

26. Showalter SL, Wang Z, Costantino CL, Witkiewicz AK, Yeo CJ, Brody JR and Carr BI: Naturally occurring K vitamins inhibit pancreatic cancer cell survival through a caspase-dependent pathway. J Gastroenterol Hepatol 25: 738-744, 2010.

27. Enomoto M, Tsuchida A, Miyazawa K, Yokoyama T, Kawakita H, Tokita H, Naito M, Itoh M, Ohyashiki K and Aoki T: Vitamin K2-induced cell growth inhibition via autophagy formation in cholangiocellular carcinoma cell lines. Int J Mol Med 20 : 801-808, 2007.

28. Miyazawa K, Yaguchi M, Funato K, Gotoh A, Kawanishi Y, Nishizawa Y, You A and Ohyashiki K: Apoptosis/differentiation-inducing effects of vitamin K2 on HL-60 cells: Dichotomous nature of vitamin K2 in leukemia cells. Leukemia 15: 1111-1117, 2001.

29. Karin M and Ben-Neriah Y: Phosphorylation meets ubiquitination: The control of NF-[kappa]B activity. Annu Rev Immunol 18: 621-663, 2000

30. Masaki T, Shiratori Y, Rengifo W, Igarashi K, Yamagata M, Kurokohchi K, Uchida N, Miyauchi Y, Yoshiji H, Watanabe S, et al: Cyclins and cyclin-dependent kinases: Comparative study of hepatocellular carcinoma versus cirrhosis. Hepatology 37: 534-543, 2003.

31. Karin $M$ and Delhase M: The I kappa B kinase (IKK) and NF-kappa B: Key elements of proinflammatory signalling. Semin Immunol 12: 85-98, 2000.
32. Holden NS, Squires PE, Kaur M, Bland R, Jones CE and Newton R: Phorbol ester-stimulated NF-kappaB-dependent transcription: Roles for isoforms of novel protein kinase C. Cell Signal 20: 1338-1348, 2008

33. Otsuka M, Kato N, Shao RX, Hoshida Y, Ijichi H, Koike Y, Taniguchi H, Moriyama M, Shiratori Y, Kawabe T and Omata M: Vitamin K2 inhibits the growth and invasiveness of hepatocellular carcinoma cells via protein kinase A activation. Hepatology 40: 243-251, 2004.

34. Liu W, Nakamura H, Yamamoto T, Ikeda N, Saito M, Ohno M, Hara N, Imanishi H, Shimomura S, Yamamoto T, et al: Vitamin $\mathrm{K} 2$ inhibits the proliferation of HepG2 cells by up-regulating the transcription of p21 gene. Hepatol Res 37: 360-365, 2007

35. Maniwa Y, Kasukabe T and Kumakura S: Vitamin K2 and cotylenin A synergistically induce monocytic differentiation and growth arrest along with the suppression of c-MYC expression and induction of cyclin G2 expression in human leukemia HL-60 cells. Int J Oncol 47: 473-480, 2015.

36. Yamamoto T, Nakamura H, Liu W, Cao K, Yoshikawa S, Enomoto H, Iwata Y, Koh N, Saito M, Imanishi H, et al: Involvement of hepatoma-derived growth factor in the growth inhibition of hepatocellular carcinoma cells by vitamin $\mathrm{K}(2)$. J Gastroenterol 44: 228-235, 2009.

37. Kaneda M, Zhang D, Bhattacharjee R, Nakahama K, Arii S and Morita I: Vitamin K2 suppresses malignancy of HuH7 hepatoma cells via inhibition of connexin 43. Cancer Lett 263: 53-60, 2008.

38. Trosko JE: The role of stem cells and gap junctions as targets for cancer chemoprevention and chemotherapy. Biomed Pharmacother 59 (Suppl 2): S326-S331, 2005.

39. Karasawa S, Azuma M, Kasama T, Sakamoto S, Kabe Y, Imai T, Yamaguchi Y, Miyazawa K and Handa H: Vitamin K2 covalently binds to Bak and induces Bak-mediated apoptosis. Mol Pharmacol 83: 613-620, 2013.

40. Shibayama-Imazu T, Sonoda I, Sakairi S, Aiuchi T, Ann WW Nakajo S, Itabe H and Nakaya K: Production of superoxide and dissipation of mitochondrial transmembrane potential by vitamin K2 trigger apoptosis in human ovarian cancer TYK-nu cells. Apoptosis 11: 1535-1543, 2006.

41. Tsujioka T, Miura Y, Otsuki T, Nishimura Y, Hyodoh F, Wada $\mathrm{H}$ and Sugihara T: The mechanisms of vitamin K2-induced apoptosis of myeloma cells. Haematologica 91: 613-619, 2006.

42. Nishimaki J, Miyazawa K, Yaguchi M, Katagiri T, Kawanishi Y, Toyama K, Ohyashiki K, Hashimoto S, Nakaya K and Takiguchi T: Vitamin K2 induces apoptosis of a novel cell line established from a patient with myelodysplastic syndrome in blastic transformation. Leukemia 13: 1399-1405, 1999.

43. Kanamori T, Shimizu M, Okuno M, Matsushima-Nishiwaki R, Tsurumi H, Kojima S and Moriwaki H: Synergistic growth inhibition by acyclic retinoid and vitamin $\mathrm{K} 2$ in human hepatocellular carcinoma cells. Cancer Sci 98: 431-437, 2007.

44. Olson JM and Hallahan AR: p38 MAP kinase: A convergence point in cancer therapy. Trends Mol Med 10: 125-129, 2004.

45. Li L, Qi Z, Qian J, Bi F, Lv J, Xu L, Zhang L, Chen H and Jia R: Induction of apoptosis in hepatocellular carcinoma Smmc-7721 cells by vitamin $\mathrm{K}(2)$ is associated with $\mathrm{p} 53$ and independent of the intrinsic apoptotic pathway. Mol Cell Biochem 342: 125-131, 2010.

46. Meek DW: Tumour suppression by p53: A role for the DNA damage response? Nat Rev Cancer 9: 714-723, 2009.

47. Muzio M, Stockwell BR, Stennicke HR, Salvesen GS and Dixit VM: An induced proximity model for caspase-8 activation. J Biol Chem 273: 2926-2930, 1998

48. Yokoyama T, Miyazawa K, Naito M, Toyotake J, Tauchi T, Itoh M, You A, Hayashi Y, Georgescu MM, Kondo Y, et al: Vitamin K2 induces autophagy and apoptosis simultaneously in leukemia cells. Autophagy 4: 629-640, 2008

49. Ide Y, Zhang H, Hamajima H, Kawaguchi Y, Eguchi Y, Mizuta T, Yamamoto K, Fujimoto K and Ozaki I: Inhibition of matrix metalloproteinase expression by menatetrenone, a vitamin K2 analogue. Oncol Rep 22: 599-604, 2009.

50. Karin M: The regulation of AP-1 activity by mitogen-activated protein kinases. Philos Trans R Soc Lond B Biol Sci 351: 127-134, 1996.

51. Zhang H, Ozaki I, Hamajima H, Iwane S, Takahashi $\mathrm{H}$, Kawaguchi Y, Eguchi Y, Yamamoto K and Mizuta T: Vitamin K2 augments 5-fluorouracil-induced growth inhibition of human hepatocellular carcinoma cells by inhibiting NF- $\mathrm{kB}$ activation. Oncol Rep 25: 159-166, 2011 
52. Funato K, Miyazawa K, Yaguchi M, Gotoh A and Ohyashiki K Combination of 22-oxa-1,25-dihydroxyvitamin $\mathrm{D}(3)$, a vitamin $\mathrm{D}(3)$ derivative, with vitamin $\mathrm{K}(2)$ (VK2) synergistically enhances cell differentiation but suppresses VK2-inducing apoptosis in HL-60 cells. Leukemia 16: 1519-1527, 2002.

53. Iguchi T, Miyazawa K, Asada M, Gotoh A, Mizutani S and Ohyashiki K: Combined treatment of leukemia cells with vitamin K2 and 1alpha,25-dihydroxy vitamin D3 enhances monocytic differentiation along with becoming resistant to apoptosis by induction of cytoplasmic p21CIP1. Int J Oncol 27: 893-900, 2005.

54. Nimptsch K, Rohrmann S, Kaaks R and Linseisen J: Dietary vitamin $\mathrm{K}$ intake in relation to cancer incidence and mortality: Results from the Heidelberg cohort of the European Prospective Investigation into cancer and nutrition (EPIC-Heidelberg). Am J Clin Nutr 91: 1348-1358, 2010.
55. Yoshiji H, Kuriyama S, Noguchi R, Yoshii J, Ikenaka Y, Yanase K, Namisaki T, Kitade M, Yamazaki M, Akahane T, et al: Amelioration of carcinogenesis and tumor growth in the rat liver by combination of vitamin $\mathrm{K} 2$ and angiotensin-converting enzyme inhibitor via anti-angiogenic activities. Oncol Rep 15: 155-159, 2006.

(c) $($ ()) This work is licensed under a Creative Common Attribution-NonCommercial-NoDerivatives 4.0 International (CC BY-NC-ND 4.0) License. 